\title{
Uso seguro de medicamentos em gestantes: construção e validação de uma cartilha educativa
}

\author{
Safe use of medicines in pregnant women: development and validation of an educational \\ booklet \\ Uso seguro de medicações en mujeres embarazadas: construcción y validación de un
folleto educativo
}

Sandna Larissa Freitas dos Santos ${ }^{1 *}$, Karla Bruna Nogueira Torres Mormino ${ }^{2}$; Hérick Hebert da Silva Alves $^{1}$, Kaléu Mormino Otoni ${ }^{2}$, Cinara Vidal Pessoa ${ }^{2}$, Aline Rebeca de Sousa Magalhães ${ }^{2}$, John Elvys Silva da Silveira², Joelson Pinheiro de Lima ${ }^{2}$, Maria Maynara Silva Souza², Eugenie Desirèe Rabelo Néri'.

\section{RESUMO}

Objetivo: Construir e validar uma cartilha educativa sobre o uso seguro de medicamentos para gestantes. Métodos: Estudo de abordagem metodológica, descritivo, transversal e quali-quantitativo, realizado duas etapas: construção da cartilha e validação por especialistas especialista na área. Resultados: A cartilha foi construída apontando respostas para algumas dúvidas sobre o uso de medicamentos, de forma dinâmica e interativa. O processo de validação foi realizado por 7 juízes especialistas, dentre eles $3(43 \%)$ enfermeiros, $2(28,5 \%)$ médicos e, $2(28,5 \%)$ farmacêuticos, sendo o perfil profissional: $2(28,5 \%)$ doutores, um doutor em obstetrícia e um em cuidados clínicos; $1(14,2 \%)$ mestre e $4(57,3 \%)$ especialistas. Obteve-se 147 respostas distribuídas em totalmente adequado $(67,34 \%, n=99)$, adequado $(30 \%, n=44)$ e $(2,72 \%, n=4)$ em parcialmente adequado. O Índice de Validade de Conteúdo (IVC) global da cartilha foi de 0,85, considerando-se validada quanto ao objetivo, estrutura e apresentação e relevância, porém alguns juízes citaram sugestões de alterações de palavras e frases, inclusão de desenhos e páginas. Conclusão: Conclui-se que a tecnologia educativa se mostrou válida quanto à aparência e ao conteúdo, se apresentando como novo material de orientação nas atividades de educação em saúde e uso na prática clínica.

Palavras-Chave: Educação em saúde, Estudos de validação, Gravidez.

\begin{abstract}
Objective: Development and validate an educational booklet about the safe use of medicines for pregnant women. Methods: This is a methodological, descriptive, cross-sectional, and quali-quantitative study, carried out in two stages: construction of the booklet and validation by specialists in the field. Results: The booklet was constructed pointing out answers to some questions about the use of medicines, in a dynamic and interactive way. The validation process was carried out by 7 expert judges, including $3(43 \%)$ nurses, $2(28.5 \%)$ doctors and $2(28.5 \%)$ pharmacists, with the professional profile: $2(28.5 \%))$ doctors, a doctor in obstetrics and one in clinical care; $1(14.2 \%)$ master and $4(57.3 \%)$ specialists. 147 responses were obtained distributed in totally adequate $(67.34 \%, n=99)$, adequate $(30 \%, n=44)$ and $(2.72 \%, n=4)$ in partially adequate. The overall Content Validity Index (CVI) of the booklet was 0.85 , considering it validated as to the objective, structure and presentation and relevance, however some judges cited suggestions for changes in words and phrases, including drawings and pages. Conclusion: It is concluded that the educational technology proved to be valid in terms of appearance and content, presenting itself as a new guiding material in health education activities and use in clinical practice.
\end{abstract}

Key words: Health education, Validation studies, Pregnancy.

1Universidade Federal do Ceará (UFC), Fortaleza - CEE. *E-mail: sandy.lary@hotmail.com

${ }^{2}$ Centro Universitário Católica de Quixadá (UNICATÓLICA), Quixadá - CE. 


\section{RESUMEN}

Objetivo: Crear y validar un folleto educativo sobre el uso seguro de medicamentos para mujeres embarazadas. Métodos: Estudio metodológico, descriptivo, transversal y cualitativo, realizado en dos etapas: construcción del folleto y validación por especialistas en el tema. Resultados: El folleto se construyó señalando respuestas a algunas preguntas sobre el uso de medicamentos, de forma dinámica e interactiva. El proceso de validación fue realizado por 7 jueces expertos, incluidos $3(43 \%)$ enfermeras, $2(28,5 \%)$ médicos y $2(28,5 \%)$ farmacéuticos, con el perfil profesional: $2(28,5 \%)$ médicos, un médico en obstetricia y otro en atención clínica; $1(14,2 \%)$ maestro y $4(57,3 \%)$ especialistas. Se obtuvieron 147 respuestas distribuidas en totalmente adecuado $(67.34 \%, n=99)$, adecuado $(30 \%, n=44)$ y $(2.72 \%, n=4)$ en parcialmente adecuado. El índice de validez de contenido (IVC) del folleto fue de 0,85 , ya que se consideró validado en cuanto al objetivo, la estructura, la presentación y la relevancia, sin embargo, algunos jueces citaron sugerencias para cambios en palabras y frases, incluidos dibujos y páginas. Conclusión: Se concluye que la tecnología educativa demostró ser válida en términos de apariencia y contenido, presentándose como un nuevo material guía en las actividades de educación para la salud y su uso en la práctica clínica.

Palabras clave: Educación para la salud, Estudios de validación, Embarazo.

\section{INTRODUÇÃO}

Na construção de cartilhas para uso em atividades de educação em saúde, há a necessidade de maximizar sua qualidade, com a análise pelos profissionais especializados na área (REBERTE LM, et al., 2012). Na perspectiva de obter instrumentos cada vez mais confiáveis e apropriados para uma específica população, o estudo de validação de conteúdo torna-se uma prática essencial (CASTRO ANP e LIMA JUNIOR EM, 2014). Estudo mostrou a relevância de um novo material de ensino nas atividades de educação em saúde para gestantes, na percepção de juízes, que são profissionais especialistas na área do estudo e gestantes (OLIVEIRA SC, et al., 2014).

A inadequação do uso de medicamentos durante a gestação, visto que essas substâncias podem gerar problemas irreversíveis ao feto por atravessar a barreira placentária, além de efeitos à gestante, é considerado um problema de saúde pública e que se configura uma prática frequente (RIBEIRO AS, et al., 2013). Estudo mostrou que prevalência para consumo de medicamentos antes e durante a gestação foi $52,1 \%$ e $84,7 \%$, respectivamente (BOMFIM CD, et al., 2017). Além da alta frequência da automedicação por esta população evidenciados em diversos estudos (BARALDO HM e YAKAWA LY, 2016; EBRAHIMI H, et al., 2017; ZEWDIE T, et al., 2018).

O uso de substâncias medicamentosas, estão dentre os principais fatores de exposição ambiental na gestação que podem ser indicadores de malformações congênitas. Esse fato foi comprovado cientificamente com o evento ocorrido com o uso da Talidomida a partir de 1950, que foi usado por gestantes para diminuir náuseas e vômitos o qual levou ao nascimento de aproximadamente 10 mil crianças com malformações congênitas (ABREU TACON, FS, et al., 2017).

A partir disso, as consequências sobre o feto em relação ao uso de medicamentos pelas gestantes são consideradas um fator de grande preocupação e estudos. No entanto, ainda é limitado pesquisas que verificam o potencial de teratogenicidade e segurança das substâncias medicamentosas e por vezes as alterações fetais só são documentadas quando ocorre algum evento nos serviços de saúde (PORTO PN, et al., 2019). Assim, prescritores acabam utilizando medicamentos mais clássicos, os quais possuem mais informações científicas (BOMFIM CD, et al., 2017).

Nesse contexto, as intervenções abordadas por meio da comunicação de conteúdos e das realizações de atividades educativas, bem como, no desenvolvimento e na avaliação dos recursos educativos, contribui na valorização da autonomia, da autoestima, da autoconfiança e da autorrealização, transformando atitudes e realidades, com abordagem do uso seguro de medicamentos no cotidiano (CASTRO ANP e LIMA JUNIOR EM, 2014; LIMA ACMACC, et al., 2017). Para as gestantes, as cartilhas educativas podem ser direcionadas 
para permitir o empoderamento e autonomia ao longo da gestação e nos primeiros meses após o parto, trazendo dicas e sugestões essenciais para que esta nova etapa da vida seja vivenciada de maneira plena e saudável para a mulher e o bebê (INDARA CB, et al. 2018).

Tendo em vista que durante a gestação, a mulher vivencia diversas transformações físicas e emocionais que requerem um cuidado especial, direcionado e específico para permitir o crescimento e o desenvolvimento saudável do feto (PORTO PN, et al., 2019). As dúvidas, curiosidades, ansiedade, insegurança e receios são comuns nesse período, enfatizando que o pré-natal constitui uma etapa de procedimentos clínicos e educativos com a necessidade de incentivar o cuidado em saúde, detectar precocemente complicações e minimizar o uso de medicamentos que possam oferecer perigo à grávida ou ao concepto (INDARA CB, et al. 2018).

Reforça-se que cartilha educativa, no formato impresso, pode ser dedicada a população com abordagens de doenças, orientações preventivas, modalidades de tratamento e autocuidado (TORRES HC, et al., 2009), que podem ser apresentadas por meio da exposição de conceitos e mensagens, perguntas e respostas ou mesmo de forma informativa. Dessa forma, o processo de aprendizado permite o paciente e os indivíduos ao seu redor uma leitura posterior, como forma de estimular as informações verbalizadas, serviço como norte de dúvidas em suas residências e auxiliando na tomada de decisões do cotidiano. Assim, a pesquisa teve como objetivo construir e validar uma cartilha educativa sobre o uso seguro de medicamentos para gestantes.

\section{MÉTODOS}

Trata-se de um estudo de abordagem metodológica, descritivo, transversal e quali-quantitativo, realizado em duas etapas: construção da cartilha e de validação por especialistas na área. A cartilha foi elaborada por Santos SLF, et al. (2019), e está disponível em http://www2.ebserh.gov.br/web/meac-ufc/cartilhas.

A primeira fase foi desenvolvida pela produção do material educativo, com uma revisão bibliográfica, contemplada pela leitura minuciosa das publicações, permitindo a categorização da temática, delimitação do conteúdo e a estruturação do material. Essa leitura resultou na seleção dos seguintes tópicos para compor a cartilha: administração, armazenamento e descarte adequado de medicamentos; evento adverso, automedicação, interação medicamentosa, intoxicação medicamentosa e uso de plantas medicinais durante a gestação. Integrou-se a proposta de uma linguagem adaptada, permitindo fácil compreensão e tornando acessível para os de pouca ou nenhuma escolaridade (FEITOZA SMS, 2015). Buscou-se a apresentação de poucas informações por páginas, dispondo de orientações compiladas da literatura, seguindo uma sequência lógica, deixando claras as condutas referentes ao uso de medicamentos de forma positiva e em sentença curta quando possível.

A seleção das ilustrações foi associada ao conteúdo, adaptando ao contexto sociocultural do público-alvo, dando ênfase à disposição das orientações com os desenhos, evitando seu uso meramente ilustrativo, com distribuição próxima à referida informação, mostrando o comportamento adequado, dando ênfase aos detalhes de cada cenário apresentado, usando símbolos e imagens para identificação do leitor, permitindo, assim, a assimilação efetiva de cada orientação. $O$ trabalho de design e diagramação das imagens foi realizado por profissional de comunicação e publicidade, com o auxílio dos programas Corel Draw Essentials $\AA$ para desenhar, o Adobe Photoshop $\AA^{\circledR}$ para colorir, e, por fim, a diagramação da cartilha e configuração das páginas no Adobe Indesign $\AA^{\circledR}$. Todas as imagens e personagens foram desenhadas para a referida cartilha.

Em seguida, percorreu-se pela validação da cartilha com os juízes, realizada mediante consulta a especialistas da área de interesse. A amostragem foi do tipo bola de neve, em que os participantes usam seu próprio julgamento para escolher outros participantes, assim, houve o recrutamento dentre os profissionais da Maternidade Escola Assis Chateaubriand (MEAC) considerados pelos que apresentaram atuação em Obstetrícia, Assistência com gestantes, com experiência em Tecnologias educativas e em Farmácia Clínica. Além disso, no processo de seleção dos juízes adotou-se o sistema de classificação de juízes em que foi utilizado os critérios de titulação acadêmica (especialização, mestrado e doutorado) e maior tempo de atuação na área. Foi disponibilizado aos participantes o convite formal, termo de consentimento livre e esclarecido, versão inicial da cartilha educativa e o instrumento para análise do material e caracterização do perfil profissional dos juízes e foi estabelecido um prazo de 15 dias para a devolução do material respondido. 
O questionário de avaliação encaminhado aos juízes especialistas foi o Suitability Assessment of Materials (SAM) adaptado de Teixeira E e Mota VMSS (2011), para avaliação da dificuldade e conveniência de materiais educativos, na qual há lista para checagem de domínios relacionados ao objetivo proposto, referindo aos propósitos da utilização do material educativo, estrutura e apresentação, refere-se a estruturação do material e relevância, em que se refere ao nível de importância do material educativo. Esse utiliza o padrão da escala tipo Likert (1- Inadequado, 2- Parcialmente Adequado, 3- Adequado, 4- Totalmente Adequado) (LIKERT R, 1932). Foi empregado o Índice de Validade de Conteúdo (IVC) para validação do instrumento, sendo desejável valor superior a 0,78 (ALEXANDRE NMC e COLUCI MZO, 2011). Os dados descritivos foram inseridos em planilha eletrônica do software Excel Microsoft Windows ${ }^{\circledR}$, versão 2010 e em seguida, transpostos para o software IBM SPSS® versão 22.0.

O estudo foi aprovado pelo Comitê de Ética em Pesquisa da Maternidade Escola Assis Chateaubriand onde a pesquisa foi realizada, sendo assegurado o cumprimento às recomendações da Resolução № 466/12 (BRASIL, 2012), recebendo parecer favorável ( $n$ ํㅜ 2.423.552).

\section{RESULTADOS}

\section{Construção da cartilha}

Após a leitura das publicações, foi compilado a temática e a dinamização da estrutura do material, em que foi construído com linguagem simples, de forma a apontar respostas para as seguintes dúvidas sobre o uso de medicamentos: Como devo tomar o medicamento? Onde devo guardar o medicamento em casa? O que fazer com os medicamentos vencidos? O que é um evento adverso? Quais os riscos da automedicação? Os fitoterápicos e preparações com plantas medicinais podem fazem mal?

O título escolhido para a cartilha foi "Uso seguro de medicamentos em gestantes" e sua elaboração teve por objetivo ofertar as informações de forma agradável, associada a imagens para aumentar a retenção do conhecimento, algumas em texto direto e outras sob a forma de jogos tais como: assinalar a conduta correta, palavras cruzadas, criptograma, caça-palavras e encontre os erros, abordando aspectos relacionados ao local de armazenamento do medicamento nas residências, conduta correta dos medicamentos vencidos, efeitos da automedicação e sobre o seguimento de consulta médica e uso de medicamentos, como uma estratégia para tornar o aprendizado agradável, interativo e dinâmico (Figura 1).

Além disso, foi disponibilizado espaço na cartilha para registros de dúvidas para serem solucionadas na consulta médica; registro de esquecimentos de dose de medicamentos para informar ao médico e ao farmacêutico, além de espaços destinados a anotação de contatos dos profissionais e do hospital. A cartilha foi apresentada em cores, formato brochura, tamanho A5, contendo 20 páginas ilustradas.

\section{Validação com juízes especialistas}

A cartilha foi validada com 7 juízes especialistas, compostos por 3 (43\%) enfermeiros, 2 (28,5\%) médicos e, $2(28,5 \%)$ farmacêuticos. O perfil profissional indicou: $2(28,5 \%)$ doutores, um doutor em obstetrícia e um em cuidados clínicos; 1 (14,2\%) mestre e 4 (57,3\%) especialistas. Com relação à ocupação atual, 4 (57,3\%) atuavam na área assistencial de ginecologia/obstetrícia, 2 (28,5\%) em farmácia clínica e $1(14,2 \%)$ na docência. Apenas $2(28,5 \%)$ juízes apresentaram experiência na elaboração de material educativo. Foram obtidas 147 respostas pelo instrumento que foram predominantemente totalmente adequado $(67,34 \%, n=99)$ e adequado (30\%, $n=44)$, com apenas 2,72\% ( $n=4)$ em parcialmente adequado (Quadro 1).

O IVC global da cartilha foi de 0,85 , considerando-se validada quanto ao objetivo, estrutura e apresentação e relevância. No entanto, alguns juízes citaram sugestões de alterações de palavras e frases, inclusão de desenhos e páginas. Todas as sugestões foram aceitas (Quadro 2). 
Figura 1 - Capa, orientações sobre administração de medicamentos e jogo didático da cartilha educativa "Uso seguro de medicamentos em gestantes". Fortaleza - CE, 2018.

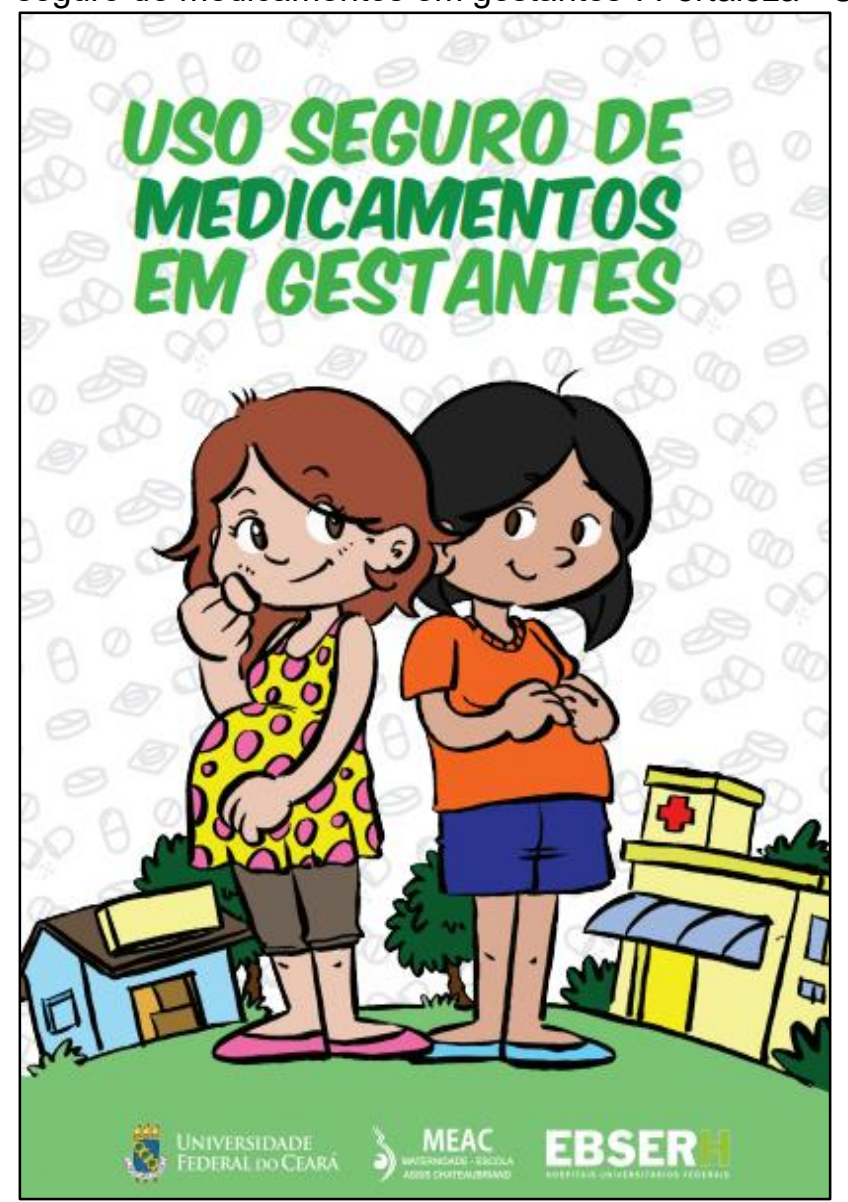

\section{COMO DEVO TOMAR MEU MEDICAMENTO?}

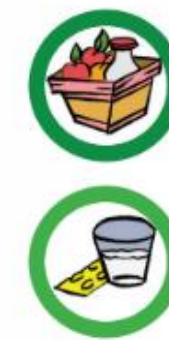

POSSO TOMAR JUNTO COM ALIMENTOS?

Sempre siga a orientação médica e do farmacêutico, cada medicamento tem sua forma adequada de ser ingerido.

\section{POSSO TOMAR COM LEITE?}

O medicamento foi estudado para ser tomado com água, outras bebidas podem interferir no efeito. Assim, água deve ser a primeira escolha.

\section{POSSO CORTAR COMPRIMIDO OU ABRIR} CÁPSULA?

Cada forma farmacêutica foi desenvolvida para beneficiar o tratamento. Pergunte ao médico ou ao farmacêutico antes de cortar comprimidos ou abrir cápsulas.

\section{POSSO TIRAR DA EMBALAGEM?}

A embalagem protege o medicamento da luz e umidade, além de permitir sua identificação, portanto mantenha os medicamentos dentro da embalagem original.

\section{3}

TESTE SEUS CONHECIMENTOS

\section{ONDE DEVO GUARDAR 0 MEDICAMENTO EM CASA?}

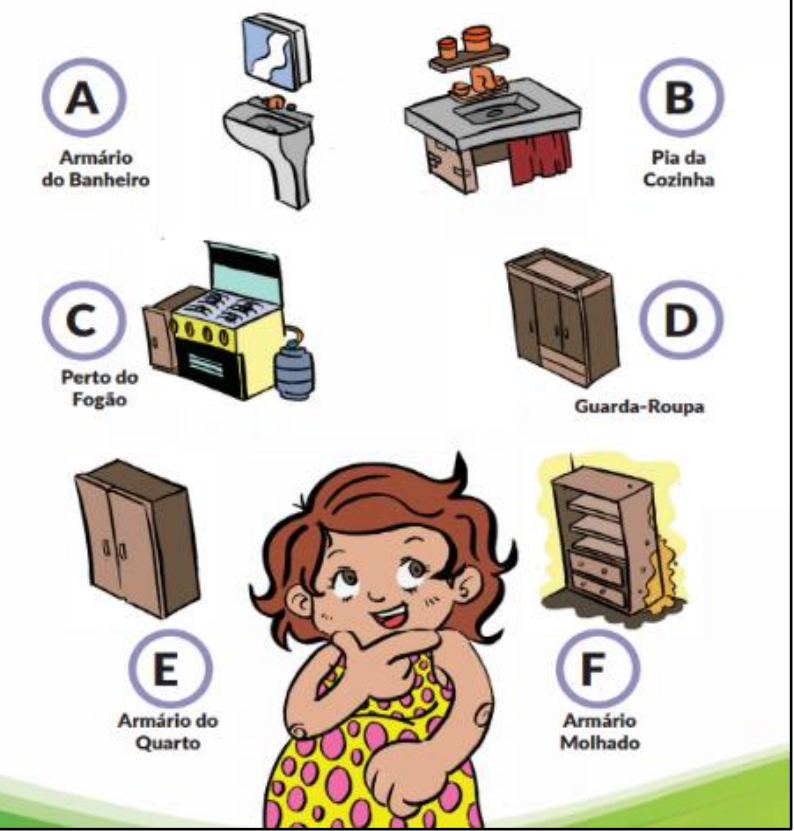

Fonte: Santos SLF, et al., 2018. 
Quadro 1 - Avaliação da cartilha educativa de acordo com os objetivos, estrutura e apresentação e relevância. Fortaleza - CE, 2018.

\begin{tabular}{|l|c|c|} 
Domínios & $\begin{array}{c}\text { Parcialmente } \\
\text { adequado }\end{array}$ & Adequado \\
\hline
\end{tabular}

\begin{tabular}{|l|l|} 
Totalmente adequado & IVC \\
\hline
\end{tabular}

1. Objetivos: Referem-se aos propósitos e metas que se deseja atingir com a utilização do material educativo.

1.1 São coerentes com as necessidades das gestantes em relação ao uso seguro de medicamentos?

1.2 Poderá promover mudança de comportamento e atitudes?

1.3 Pode circular no meio científico na área da Obstetrícia?

\begin{tabular}{|l|l|l|}
\hline 1 & 6 & 1,00 \\
\hline 3 & 4 & 1,00 \\
\hline 1 & 6 & 1,00 \\
\hline
\end{tabular}

\section{Estrutura e apresentação: Refere-se a forma de apresentar as orientações. Isto inclui sua organização geral, estrutura, estratégia de apresentação,} coerência e formatação.

2.1 O material educativo é apropriado para orientação de gestantes em relação ao uso de medicamentos?

2.2 As mensagens estão apresentadas de maneira clara?

2.3 As informações estão apresentadas de forma objetiva?

2.4 As informações apresentadas estão cientificamente corretas?

2.5 Há uma sequência lógica do conteúdo proposto?

2.6 O material está adequado ao nível sociocultural do público-alvo proposto?

2.7 As informações são bem estruturadas gramaticalmente (concordância e ortografia)?

2.80 estilo de redação corresponde ao nível de conhecimento do público-alvo?

2.9 Informações da capa, contracapa, agradecimentos e/ou apresentação são coerentes?

2.10 As ilustrações são expressivas e suficientes?

2.11 O número de páginas está adequado?

2.12 O tamanho do título e dos tópicos está adequado?

2.13 A visualização em meio eletrônico é agradável?

\begin{tabular}{|l|l|l|}
\hline & 6 & 1,00 \\
\hline 5 & 2 & 1,00 \\
\hline 3 & 4 & 1,00 \\
\hline 4 & 3 & 1,00 \\
\hline 1 & 5 & 0,85 \\
\hline 6 & & 0,85 \\
\hline 2 & 4 & 0,85 \\
\hline 5 & 1 & 0,85 \\
\hline 1 & 6 & 1,00 \\
\hline & 7 & 1,00 \\
\hline 3 & 4 & 1,00 \\
\hline 2 & 5 & 1,00 \\
\hline 2 & 5 & 1,00 \\
\hline
\end{tabular}

3. Relevância: Refere-se ao grau de importância do material educativo apresentado para a promoção do uso seguro e racional de medicamentos.

3.1 Os temas retratam os aspectos chaves que devem ser reforçados?

3.20 material propõe a paciente adquirir conhecimento quanto ao uso seguro e racional de medicamentos durante a gestação?

3.3 O material aborda os assuntos necessários para a prevenção de complicações relacionadas ao uso equivocado de medicamentos e reações adversas?

3.4. Está adequado para ser utilizado por qualquer profissional da área da saúde em suas atividades educativas?

Fonte: Santos SLF, et al., 2018.

\begin{tabular}{|l|l|l|}
\hline 2 & 7 & 1,00 \\
\hline 1 & 6 & 1,00 \\
\hline 1 & 6 & 1,00 \\
\hline
\end{tabular}

REAS/EJCH | Vol.Sup.n.49 | e3274 | DOI: https://doi.org/10.25248/reas.e3274.2020

Página 1 de 12 


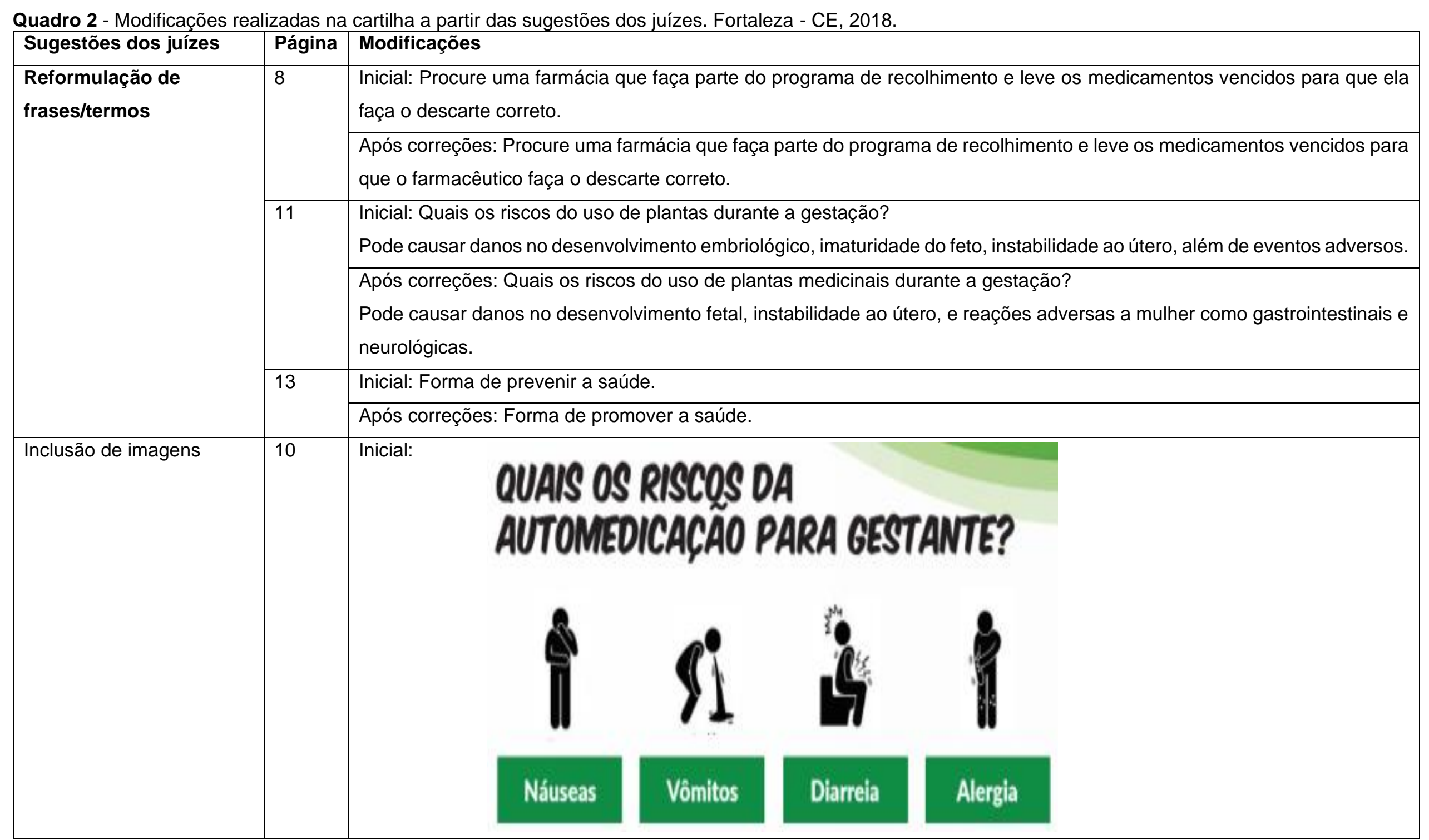




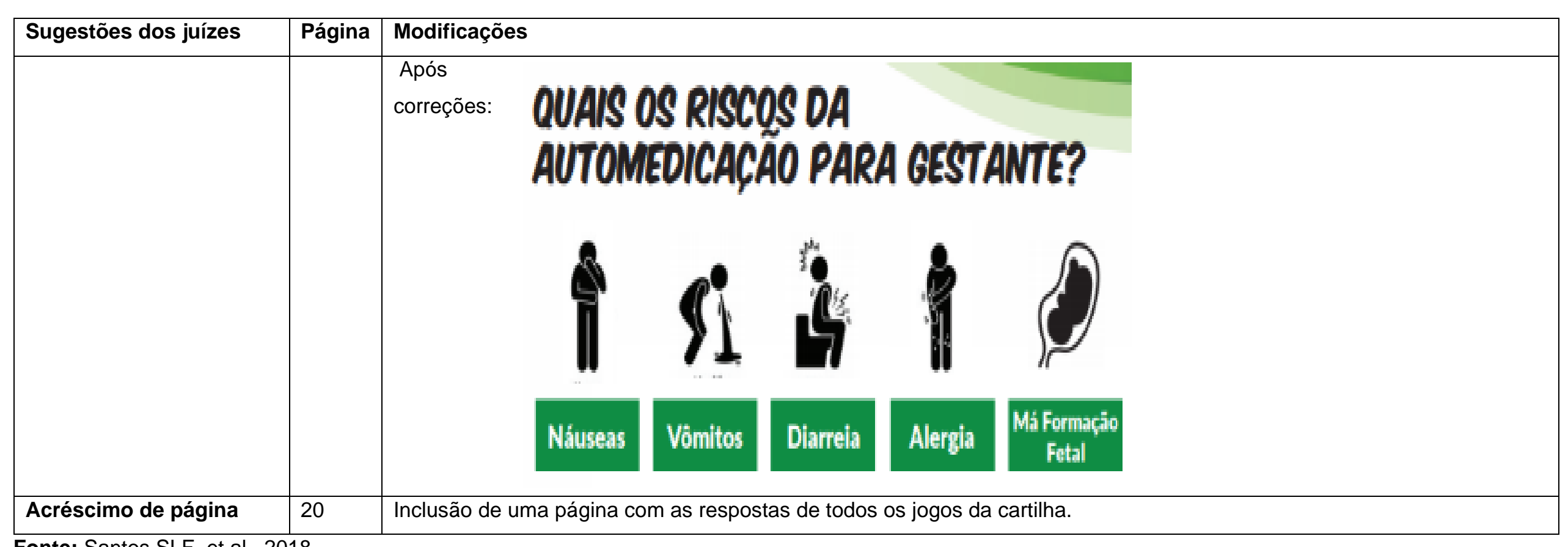

Fonte: Santos SLF, et al., 2018. 


\section{Validação com juízes especialistas}

A cartilha foi validada com 7 juízes especialistas, compostos por $3(43 \%)$ enfermeiros, 2 (28,5\%) médicos e, $2(28,5 \%)$ farmacêuticos. O perfil profissional indicou: $2(28,5 \%)$ doutores, um doutor em obstetrícia e um em cuidados clínicos; 1 (14,2\%) mestre e 4 (57,3\%) especialistas. Com relação à ocupação atual, 4 (57,3\%) atuavam na área assistencial de ginecologia/obstetrícia, 2 (28,5\%) em farmácia clínica e 1 (14,2\%) na docência. Apenas $2(28,5 \%)$ juízes apresentaram experiência na elaboração de material educativo. Foram obtidas 147 respostas pelo instrumento que foram predominantemente totalmente adequado $(67,34 \%, n=99)$ e adequado (30\%, $n=44)$, com apenas 2,72\% ( $n=4)$ em parcialmente adequado (Quadro 1).

O IVC global da cartilha foi de 0,85 , considerando-se validada quanto ao objetivo, estrutura e apresentação e relevância. No entanto, alguns juízes citaram sugestões de alterações de palavras e frases, inclusão de desenhos e páginas. Todas as sugestões foram aceitas (Quadro 2).

Dos 7 participantes $4(57,3 \%)$ apresentaram comentários sobre a cartilha, relacionados ao conteúdo, ilustrações e a viabilidade da aplicação na prática clínica dos serviços de saúde (Quadro 3). Além disso, os juízes avaliadores forneceram elogios sobre disposição das informações e da cartilha em geral, destacando a importância de estudos que fornecem a construção e a validação de materiais.

Quadro 3 - Comentários dos juízes especialistas sobre a cartilha "Uso seguro de medicamentos em gestantes" - Fortaleza-CE, 2018.

\begin{tabular}{|l|l|}
\hline Categoria & Comentários \\
\hline Linguagem & $\begin{array}{l}\text { No geral está ótima, sugiro apenas rever algumas palavras, para ficar na linguagem do } \\
\text { nivel das gestantes e para facilitar a compreensão. }\end{array}$ \\
\hline Aplicabilidade & $\begin{array}{l}\text { Material muito bom para ser usado em gestantes em geral, muito útil para os } \\
\text { profissionais de saúde usarem em atividades educativas. }\end{array}$ \\
\hline Ilustrações & $\begin{array}{l}\text { Achei interessantes os desenhos, bem elaborados e pensados de forma adequadas às } \\
\text { orientações. }\end{array}$ \\
\cline { 2 - 2 } & $\begin{array}{l}\text { Os desenhos estão bem apresentados, as orientações estão dispostas pertinentes e } \\
\text { apropriadas à temática. }\end{array}$ \\
\hline
\end{tabular}

Fonte: Santos SLF, et al., 2018.

\section{DISCUSSÃO}

O processo de ajuste do material educativo com a análise dos juízes é considerado uma etapa essencial para elevar a qualidade do conteúdo apresentado em Tecnologia educacionais, com uma metodologia científica e tornando-a apta sua utilização nas atividades de educação em saúde (ECHER IC, 2005). Estudos também apresentam essa etapa consistente na validação, em que sugestões, reformulações e a exclusões de informações, adequação da linguagem de acordo com o público-alvo, favorecem o aperfeiçoamento do material (COSTA PB, et al., 2013; LIMA ACMACC, et al., 2017).

Ações de educação em saúde, mediadas por tecnologia educacional no formato impresso, tipo cartilha, dispõe como uma forma de cuidado, promoção da saúde e prevenção de agravos, proporcionando potencialidades dos pacientes. Nisso, atrai um sistema de relação profissional e pacientes, convergência para transformação de realidades, tornando dinâmico e atrativo o aprendizado (MEIRELLES RMS, et al., 2017).

A tecnologia educacional, usada amplamente em capacitações de profissionais e orientações educativas com a população, tem sua importância por abordar o conjunto de dados com embasamentos científicos que envolvem o processo educacional. Tem-se nesse contexto, como uma metodologia amplamente empregada frequentemente na rotina dos serviços de saúde, funcionando como veiculador de conhecimentos, visto que a busca dos profissionais de saúde é de estratégias inovadoras que ofereçam suporte para intervenções positivas na comunidade e favoreçam o bem-estar social, bem como à sensibilização do público, nos mais diversos enfoques de promoção da saúde (LESSA LP, et al., 2018).

Sobre a sensibilização da população, Benevides JL, et al. (2016) trazem a ideia de que na prática diária nos serviços de saúde os materiais educativos impressos, como manuais, folhetos, folders, livretos, álbum 
seriado e cartilhas se tornam estratégias eficazes, favorecendo a disseminação de conhecimento entre pacientes e cuidadores, seja a respeito de uma doença ou na adesão a um tratamento, além de atuar como uma metodologia que gera poder de decisão dos pacientes.

Lopes MVO e Fernandes AFC (2014) apresentam que os materiais educativos impressos têm sido utilizados como ferramenta de educação em saúde para facilitar o conhecimento, esclarecer mitos e tabus relacionados a uma temática social, cultura, política e aspectos relacionado a saúde humana. O uso de material impresso auxilia como objetivo orientações, além de ser um recurso que a pessoa também poderá utilizar na ausência do profissional capacitado.

A estratégia de implementação dos jogos atuou como um espaço privilegiado para a promoção da aprendizagem, influenciando positivamente no interesse e na motivação, facilitando a assimilação de conceitos pela estimulação do processo cognitivo, permitindo a expressão de opiniões, esclarece conceitos, reforçando e suplementando as orientações. As gestantes avaliadoras analisaram o uso desse recurso como forma de garantir a aprendizagem significativa do conteúdo, por meio da maior interação do público com o material (MEIRELLES RMS, et al., 2017).

D'Avila CG, et al., (2018) numa validação de um jogo educativo para gestantes sobre boas práticas de trabalho de parto, verificaram a importância da instrução com o uso de imagens associado às orientações verbais, construindo a ideia do instrumento como autoexplicativo, além do diferencial da superação da barreira de linguagem. Benevides JL, et al. (2016) evidenciaram que a educação por meio de jogos pode ser uma metodologia crucial para construção do conhecimento, que retira de linha o aprendizado monótono e rotineiro.

No contexto de abordagem na Atenção Primária a Saúde, Indara CB, et al. (2018) apresentaram um estudo de validação de uma tecnologia educativa direcionada a gestantes, com os juízes profissionais e usuárias, mostrando que o material pode ser usado como apoio complementar e representar um guia prático, de rápido acesso e manuseio para sanar as dúvidas recorrentes da gravidez. A abordagem de desenhos e figuras em materiais educativos também é destacado em outros estudos (CASTRO ANP e LIMA JUNIOR EM, 2014; CORDEIRO LI, et al., 2017; MOURA IH, et al., 2017). Para Vasconcelos SS, et al. (2018) há a necessidade de aliar o conteúdo escrito com ilustrações para melhor fixação das informações transmitidas e que o Layout e Design devem ser criados de forma a fixar a atenção do leitor, além de associar com uma linguagem adaptada a cada perfil de público.

A temática abordada na cartilha constitui uma abordagem significativa, destacando o alto consumo de medicamentos em gestantes, além da crescente ocorrência de automedicação (SANTOS SLF, et al., 2018) e pela necessidade de instrumentos que norteiem essa prática em ambientes fora do hospital (REBERTE LM, et al., 2012). O principal objetivo do material foi proporcionar a disseminação da temática por meio de estratégia interativa e dinamizada, a fim de garantir a atenção, visto que é direcionado a mulheres adultas.

A estruturação da sistematização da cartilha se deu por meio de dúvidas e questionamentos que surgem rotineiramente pelas gestantes, quanto ao uso de medicamentos, que por vezes, não são esclarecidas em consultas médicas. A busca pela associação dos desenhos e orientações dispostas de forma adequadas, foi provocada por estudos (CASTRO ANP e LIMA JUNIOR EM, 2014; OLIVEIRA SC, et al., 2014), em que constataram na validação com juízes especialistas, o conteúdo associado as ilustrações motivam a leitura pelo público-alvo.

Quanto ao perfil dos profissionais avaliadores do material, com maior prevalência de 3 (43\%) enfermeiros e $4(57,3 \%)$ especialistas, condiz com dados encontrados em outras pesquisam como citam Benevides JL, et al. (2016) e Oliveira SC, et al. (2014). Além disso, os autores destacam a importância da participação de profissionais farmacêuticos, em que garantem orientação qualificada sobre o uso de medicamentos e os riscos inerentes a essa prática.

Enfatiza-se que no processo de validação pelos juízes, priorizou-se pela multidisciplinaridade das categorias profissional dos juízes, visando a multiplicidade do conhecimento, ampliação de vivências profissionais e unificação dos saberes especializados da área da saúde, a fim de permitir uma avaliação ampliada da temática abordada pelo material. Dessa mesma forma, outras pesquisas também ressaltam esse 
aspecto, deixando claro que a atuação de uma equipe multiprofissional possibilita diferentes julgamentos e visões sobre o mesmo tema e garantia da diversificação da qualidade do material (LIMA ACMACC, et al., 2017; CORDEIRO LI, et al., 2017; VASCONCELOS SS, et al., 2018).

Apesar de o IVC global ter-se apresentado adequado no presente estudo $(0,85)$ os juízes apresentaram sugestões que resultaram em mudanças relevantes na cartilha. Os autores contextualizam esse indicador como uma estratégia de mensuração da qualidade e efetividade das informações contidas no material e na repercussão que pode gerar no cotidiano da população, além disso pode ser um fator norteador de aprovação pelos avaliadores.

Comparado a esse resultado, outros estudos também com validação com juízes especialistas apresentaram IVC semelhantes (LIMA ACMACC, et al., 2017; CASTRO ANP e LIMA JUNIOR EM, 2014; MOURA IH, et al., 2017). Assim, Benevides JL, et al. (2016) encontraram na pesquisa que o IVC representa um indicador que podem mensurar a validade do material de acordo com o conhecimento dos avaliadores e as perspectivas de sua aplicação nos serviços.

Os comentários dos avaliadores concordaram com a aplicabilidade em ações educativas, demonstrando alta confiabilidade no conteúdo e sua concordância com as ilustrações e na facilidade da compreensão das orientações dispostas. Comparando esses resultados, o estudo de Moura IH, et al. (2017) apresentam que a disponibilidade de comentários para os juízes é um campo aberto que permite a inserção de elogios e sugestões de melhoria, que podem ser eficazes para as transformações do material em questão.

Destaca-se que apesar da multidisciplinaridade dos juízes em que a disposição diferenciada de olhares ampliados, concretizam o trabalho em equipe, com visão de cada categoria na mesma temática, permitindo vincular a competência, habilidade e experiência dos profissionais em um mesmo propósito, o estudo apresenta como limitação a ausência da validação com profissionais da área de publicidade e comunicação e por não ser possível verificar o impacto do uso de medicamentos frente ao material educativo. Nesse contexto, o estudo permeia a necessidade de pesquisas posteriores com o público alvo da cartilha educativa, que validem a utilização nos serviços de saúde e avaliem o impacto no uso seguro de medicamentos por gestantes.

\section{CONCLUSÃO}

A tecnologia educativa mostrou-se válida quanto à aparência e ao conteúdo, com potencial de utilização na prática clínica, tornando evidente a relevância e se apresentando como novo material de orientação nas atividades de educação em saúde, com o objetivo de motivar a gestante ao uso seguro de medicamentos. Com a validação com os juízes profissionais a cartilha apresentou algumas características positivas como: informativa, de linguagem clara e de fácil compreensão, com imagens ilustrativas e apresentando de forma interativa e dinâmica. Percebe-se que além da utilização pelos profissionais nos serviços de saúde, destacase o uso pela própria gestante, como processo de autocuidado e empoderamento principalmente por se estruturar no formato de responder questionamentos do cotidiano. Destaca-se que a validação com as gestantes será fruto de outra publicação. A cartilha está disponível no site da Maternidade Escola Assis Chateaubriand e será utilizada em pesquisas posteriores a fim de avaliar o impacto do uso de medicamentos durante a gestação com vista na aplicação do material nas ações educativas.

\section{REFERÊNCIAS}

1. ABREU TACON, FS, et al. Medicamentos e gravidez: Influência na morfologia fetal. Rev. Educ. Saúde. 2017; 5(2): 105-111.

2. ALEXANDRE NMC, COLUCI MZO. Validade de conteúdo nos processos de construção e adaptação de instrumentos de medidas. Ciência \& Saúde Coletiva, Rio de Janeiro, 2011; 16(7): 3061-3068.

3. BARALDO HM, YAKAWA LY. Automedicação entre gestantes assistidas em serviço público de saúde no município de Floresta, Paraná. Revista UNINGÁ, 2016; 25(3):31-35.

4. BENEVIDES JL, et al. Desenvolvimento e validação de tecnologia educacional para atendimento de úlcera venosa. RevEscEnferm USP. 2016 Apr;50(2):309-16. doi: 10.1590/S0080-623420160000200018.

5. BENEVIDES JL, et al. Construção e validação de tecnologia educativa sobre cuidados com úlcera venosa. Rev Esc Enferm USP [Internet]. 2016 [acesso em 2018 Ago 15];50(2):309-16. 
6. BOMFIM CD, et al. Utilização de medicamentos antes e durante a gestação: prevalência e fatores associados. Cad. Saúde Pública [Internet]. 2017 [cited 2019 Jan 02]; 33(2): e00126215.

7. BRASIL. Conselho Nacional de Saúde, Resolução ํㅜ66 de 12 de dezembro de 2012.

8. CASTRO ANP, LIMA JUNIOR EM. Desenvolvimento e validação de cartilha para pacientes vítimas de queimaduras. RevBras Queimaduras. 2014;13(2):103-13.

9. COSTA PB, et al. Development and validation of educational manual for the promotion of breastfeeding. Rev Rene. 2013; 14(6):1160-7.

10. CORDEIRO LI, et al. Validação de cartilha educativa para prevenção de HIV/Aids em idosos. Rev Bras Enferm [Internet]. 2017 jul-ago;70(4):808-15.

11. D'AVILA CG, et al. Construção e validação de jogo educativo para gestantes. Escola Anna Nery, 2018; 22(3).

12. ECHER IC. The development of handbooks of health care guidelines. Ver LatAm Enfermagem. 2005; 13(5): 754-7

13. EBRAHIMI $\mathrm{H}$, et al. Self-medication and its risk factors among women before and during pregnancy.Pan AfrMed J. 2017 Jul 7;27:183. doi: 10.11604/pamj.2017.27.183.10030. eCollection 2017.

14. FEITOZA SMS. Construção e validação de uma cartilha educativa para orientação das mães sobre os cuidados póstransplantes cardíacos pediátrico. 2015. 158 f. Dissertação (Mestrado) - Faculdade de Farmácia, Odontologia e Enfermagem, Universidade Federal do Ceará, Fortaleza, 2015.

15. INDARA CB, et al. Tecnologia Educativa para Gestantes: Construção e Avaliação de Cartilha. Convención Internacional de Salud, Cuba Salud; 2018, 11(2): 1-8.

16. LESSA LP, et al. Construção de uma Cartilha Sobre Educação no Trânsito para AdolescenteS. Rev enferm UFPE on line., Recife, 12(10):2737-42, out., 2018.

17. LIMA ACMACC, et al. Construção e Validação de cartilha para prevenção da transmissão vertical do HIV. Acta Paul Enferm. 2017; 30(2):181-9

18. LIKERT, R. A technique for the measurement of attitudes. Archives of Psychology. n. 140, p. 44-53, 1932.

19. LOPES MVO, FERNANDES AFC. Development and validation of an educational booklet for healthy eating during pregnancy. Rev Latino-Am Enfermagem [Internet]. 2014 [cited 2017 Jan 17];22(4):611-20. Available from: http://www.scielo.br/pdf/rlae/ v22n4/0104-1169-rlae-22-04-00611.pdf.

20. MEIRELLES RMS, et al. Jogos sobre Educação em Saúde: Limites e Possibilidades. Enseñanza De Las Ciencias, Extraordinario, p. 5079-5084, 2017.

21. MOURA IH, et al. Construction and validation of educational materials for the prevention of metabolic syndrome in adolescents. Rev Lat Am Enfermagem. 2017 Oct 5;25: e2934. doi: 10.1590/1518-8345.2024.2934.

22. OLIVEIRA SC, et al. Construção e validação de cartilha educativa para alimentação saudável durante a gravidez.Rev. Latino-Am. Enfermagem Artigo Original jul.-ago. 2014;22(4):611-20.

23. PORTO PN, et al. Fatores associados ao envolvimento de gestantes com álcool e outras drogas. Revista Eletrônica Acervo Saúde 2019; 11(2): 1-8.

24. REBERTE LM, et al. O processo de construção de material educativo para a promoção da saúde da gestante. Rev. Latino-Am. Enfermagem, 2012; 20(1).

25. RIBEIRO AS, et al. Risco potencial do uso de medicamentos durante a gravidez e a lactação, Infarma - Ciências Farmacêuticas, 2013; 25(1): 62-7.

26. SANTOS SLF, et al. Uso seguro de medicamentos em gestante. Fortaleza: Universidade Federal do Ceará (UFC), Maternidade-Escola Assis Chateaubriand, 2019. Disponível em: http://www2.ebserh.gov.br/web/meac-ufc/cartilhas

27. SANTOS SLF, et al. Automedicação em gestantes de alto risco: foco em atenção automedicação. Rev Med UFC. 2018;58(3):36-43.

28. SOUSA CS, TURRINI RNT. Construct validation of educational technology for patients through the application of the Delphi technique. Acta Paul Enferm. 2012;25(6):990-6.

29. TEIXEIRA E, MOTA VMSS. Educação em saúde: Tecnologias Educacionais em Foco. Série educação em saúde. São Caetano do Sul (SP): Difusão Editora, 2011; 2.

30. TORRES HC, et al. O processo de elaboração decartilhas para orientação do autocuidado no programa educativo em Diabetes. RevBrasEnferm. 2009;62(2):312-6.

31. VASCONCELOS SS, et al. Validação de uma Cartilha Sobre a Detecção Precoce do Transtorno do Espectro Autista. Rev Bras Promoç Saúde, 31(4): 1-7, out./dez., 2018.

32. ZEWDIE T, et al. Self-medication during pregnancy and associated factors among pregnant women in Goba town, southeast Ethiopia: a community based cross sectional study. BMC Res Notes. 2018 Oct 10;11(1):713. doi: 10.1186/s13104-018-3821-8. 